\title{
PATHOPHYSIOLOGY, TYPES, ITS MEDIATORS \& TREATMENT STRATEGIES OF ALZHEIMER'S DISEASE: A SHORT REVIEW
}

\author{
Puneet Singh*, Yogesh Sharma, Ashutosh Sharma
}

Jaipur College of Pharmacy ISI - 15 RIICO Institutional Area, Tonk Rd, Sitapura, Jaipur, Rajasthan 302022

Article Info: Received 3 January 2020; Accepted 6 February 2021

DOI: https://doi.org/10.32553/jbpr.v10i1.835

Corresponding author: Puneet Singh

Conflict of interest statement: No conflict of interest

\begin{abstract}
Cognition in an extensive sense means information dispensation. It denotes a relatively high level of processing of definite information including thinking, memory, perception, incentive, skilled arrangements and language. Learning is the most attribute attributes of the man and also of higher animals. Learning is definite as the skill to alter behavior on the basis of understanding. We know that some reminiscences last for only a few seconds, whereas others last for hours, days, months or year. Dementia is generally defined as the loss of logical abilities (medically called cognitive dysfunction) of sufficient capacity to interfere with social or occupational functioning. People with DLB have some of the symptoms common in Alzheimer's, but are more likely than people with Alzheimer's to have initial or early symptoms such as sleep disturbances, well-formed visual hallucinations, and muscle rigidity or other parkinsonian movement features. The signature lesions in AD are neuritic plaques and neurofibrillary tangles (NFTs) to be found in the cortical areas and medial temporal lobe structures of the brain.
\end{abstract}

Keywords: Alzheimer, Neurofibrillary tangles, Neuritic plaques, Dementia, Temporal lobe

\section{Introduction}

Cognitive dysfunction, a major health problem in $21^{\mathrm{st}}$ century one of the most functionally demoralizing characteristic of many neuropsychiatric disorders and neurodegenerative disorder such as Schizophrenia, AD dementia, cerebrovascular impairment, head grievance, and parkinsonism (Ingole et al., 2008).

Dementia is a brain disorder that gravely affects a person's capacity to carry out daily behavior. The most widespread form of dementia surrounded by older people is $\mathrm{AD}$, which to begin with involves the parts of the brain that control contemplation, memory, and language, ending with ruthless brain damage (Joshi et al., 2007).

Cognition in a extensive sense means information dispensation. It denotes a relatively high level of processing of definite information including thinking, memory, perception, incentive, skilled arrangements and language. The hippocampus contains the neural circuitry necessary for cognitive functions such as learning and memory (Tripathi, 2006).

The memory is the most significant functions of the brain. Memory is the process by which organisms are able to confirmation their experiences and uses this in order to adapt their responses to the environment. Hence it is vital for survival. Central cholinergic system is well thought-out as the most important neurotransmitter involved in regulation of cognitive functions. Impaired cognitive functions are the major features of $\mathrm{AD}$. Presence of acetylcholine within the neocortex is adequate to ameliorate learning deficits and refurbish memory (Kanwal et al., 2010).

\section{Learning and Memory:}

Learning is the most attribute attributes of the man and also of higher animals. Learning is definite as the skill to alter behavior on the basis of understanding (Jain, 2007). Memory is out of the ordinary facility of brain which retains the events 
developed during the development of learning and both are mediated by nervous system (Chatterjee, 1997). Once memories have been stored in the brain, it becomes the part of brain progression mechanism when it will bring to mind in future (Guyton and Hall, 2006). Learning and memory are intimately linked, all learning involves memory but all memory not involves to learning (Bijlani, 2004). Sensory information acknowledged through the eyes, ears, and other senses is registered very briefly in short-term or iconic memory (Greek; Iconic $=$ Image). Some residue of information from the iconic memory persists for a few second in short term memory (Windhorst, 1996). Declarative memory (or, explicit memory) affords the aptitude for wide awake recollections about facts and events. This is the kind of memory that is usually referred to when the terms "memory" or "remembering" are used in commonplace language. Declarative memory can be contrasted with non declarative (or implicit) memory, a mixed collection non mindful ability that includes the learning of skills and habits, priming, and some forms of usual conditioning. In these cases, understanding cumulates in behavioral change, but without affording access to any memory content. The variation between declarative and non declarative memory is elementary, because it has turned out that different kinds of memory are supported by diverse brain systems.

\section{Types of Memory}

We know that some reminiscences last for only a few seconds, whereas others last for hours, days, months or year. For the purpose of discussing these, let us use a common classification of memories that divide memories into:

Short term memory: Includes memories that last for seconds or most minutes unless they are transformed into longer term memories.

Intermediate long term memories: Which last for days to weeks but then fade away?

Long term memory: Which once stored, can be recalled up to years or even a life time later.

Working memory: Includes mainly short term memory that is used during the course of cerebral reasoning but is finished as each stage of the problem is resolved (Guyton and Hall, 2006).

Memories are frequently classified according to the type of information that is stored. One of these classifications divides memory into declarative memory and non declarative memory, as follows:

\section{Declarative memory:}

It includes memory for facts and proceedings and depends on the integrity of the hippocampus and related arrangement.

\section{Non Declarative memory:}

It refers to a mixed collection of take apart learning and memory abilities where piece changes but restricted of affording access to the experiences that caused the change (Zola \& Squire, 1990).

\section{Dementia:}

Dementia is generally defined as the loss of logical abilities (medically called cognitive dysfunction) of sufficient capacity to interfere with social or occupational functioning (Shivakumar et al., 2011). It can result from an assortment of diseases that cause damage to brain cells. There are many poles apart types of dementia, each with its own cause and symptoms. Increasing substantiation from long term epidemiological observation and autopsy studies suggests that many people have brain abnormalities associated with more than one category of dementia (Alzheimer's and Dementia, 2013).

\section{Types of dementia:}

\section{Alzheimer's disease:}

Most common type of dementia; accounts for a predictable 60 to 80 percent of cases.

Involvedness remembering names and recent events is often an early clinical symptom; apathy and depression are also often early symptoms.

Later symptoms embrace impaired opinion, disorientation, confusion, behavior changes and difficulty speaking, swallowing and walking.

New criteria and guidelines for diagnosing Alzheimer's were proposed in 2011. They recommend that Alzheimer's disease be measured a disease that begins well before the enlargement of symptoms.

Hallmark brain abnormalities are deposits of the protein splinter beta-amyloid (plaques) and twisted strands of the protein tau (tangles) as well as verification of nerve cell smash up and death in the brain.

\section{Vascular dementia:}

Previously recognized as multi-infarct or poststroke dementia, vascular dementia is less widespread as a sole cause of dementia than is Alzheimer's disease. 
Impaired judgment or ability to create plans is more likely to be the initial symptom, as opposed to the memory loss often associated with the initial symptoms of Alzheimer's.

Vascular dementia occurs since of brain injuries such as microscopic bleeding and blood vessel obstruction.

The location of the brain injury determines how the individual's thinking and physical implementation is affected.

\section{Dementia with Lewy bodies (DLB):}

People with DLB have some of the symptoms common in Alzheimer's, but are more likely than people with Alzheimer's to have initial or early symptoms such as sleep disturbances, well-formed visual hallucinations, and muscle rigidity or other parkinsonian movement features.

Lewy bodies are uncharacteristic aggregations (or clumps) of the protein alpha-synuclein. When they build up in a part of the brain called the cortex, dementia can result. Alpha-amyloid also aggregates in the brains of people with Parkinson's disease, but the aggregates may come into view in a pattern that is different from DLB.

The brain changes of DLB alone can grounds dementia, or they can be present at the same time as the brain changes of Alzheimer's disease and/or vascular dementia, with each entity contributing to the development of dementia. When this happens, the individual is said to have "mixed dementia."

\section{Front temporal lobar degeneration (FTLD):}

Includes dementias such as behavioral substitute FTLD, primary progressive aphasia Pick's disease and progressive supranuclear palsy.

\section{Mixed dementia:}

Characterized by the characteristic abnormalities of Alzheimer's and another type of dementia most commonly, vascular dementia, but also other types, such as DLB.

Recent studies recommend that mixed dementia is more common than previously thought.

\section{Anatomy and Physiology Associated with Dementia:}

All dementias can be categorized as one of two patterns of brain worsening: cortical or sub cortical. Cortical types of dementia are characterized by marked memory warfare. AD is a result of cortical commotion but finally affects all lobes to some degree26. Magnetic reverberation imaging (MRI) has shown that the disease apparently occurs in different areas of the brain as it progresses (Scahill et al., 2002). Sub cortical disorders often have associated motor disabilities.

The whole brain is involved in the process of memory. It is believed that the hippocampus (located deep in the brain above the brain stem) and the amygdala (situated under the temporal lobe) are critical to the formation, storage, and retrieval of memory.

The hippocampus, connected by afferent pathways to sensory areas of the cortex, is guilty for the acquisition and temporary storage of declarative memory. Declarative memory enables folks to organize their world. For example, one learns the route to work and after traveling the route a few times is able to get there even though there may be a alternative route. The hippocampus maintains the directory for all of these memories so when they are needed they can be recalled. Individuals with mutual loss of the hippocampus can only register incoming stimuli until the next stimulus arrives. Memories cannot be called up when needed, such as during learning experiences (Shapira et al., 1986).

The neurotransmitter that features most prominently in $\mathrm{AD}$ is acetylcholine. Dysfunction and reduction of nicotinic acetylcholine receptors is linked to adverse cognitive and neurodegenerative effects (Oddo \& Laferla, 2006).

\section{Pathophysiology of Alzheimer Disease}

The signature lesions in $\mathrm{AD}$ are neuritic plaques and neurofibrillary tangles (NFTs) to be found in the cortical areas and medial temporal lobe structures of the brain. Along with these lesions, deterioration of neurons and synapses, as well as cortical atrophy, occurs. Plaques and NFTs may also be in attendance in other diseases, even in normal aging, but there is a much higher attentiveness of plaques and NFTs in patients with $\mathrm{AD}$. The state of affairs in which these lesions lead to the clinical picture of $\mathrm{AD}$ remain unclear. Several mechanisms have been proposed to explain these changes in the brain, including $\beta A P$ aggregation and deposition leading to the arrangement of plaques; hyperphosphorylation of tau protein leading to NFT development; inflammatory processes; dysfunction of the neurovasculature; oxidative stress; and mitochondrial dysfunction.

\section{Inflammatory Mediators:}

Inflammatory or immunologic paradigms are often viewed as a corollary of the amyloid cascade proposition. Certainly, brain amyloid deposition acquaintances with local inflammatory and 
immunologic alterations. The inflammatory/ immunologic hypotheses argue that although $\beta A P$ may have direct neurotoxicity, at least some of its toxicity might actually be an indirect outcome of a $\beta$-AP protofibril-induced microglia activation and astrocyte recruitment. This inflammatory response may represent a confront to clear amyloid statement. However, it is also connected with release of cytokines, nitric oxide, and other radical species, and complement factors that can both injure neurons and encourage continuing inflammation. Indeed, levels of multiple cytokines and chemokines are elevated in $\mathrm{AD}$ brains, and certain pro inflammatory gene polymorphisms are reported to be associated with $\mathrm{AD}$ (Dipiro et al., 2008).

The first and most extensive pathological change in Alzheimer's disease is the accumulation of betaamyloid peptides into amorphous deposits. The deposition induces an inflammatory response and consequently an acute phase response involving IL1 and IL-6 in microglial cells of the brain parenchyma and TGF-betal in perivascular astrocytes. The cytokines trigger liberate of various secretory products from the surrounding astrocytes that catalyze the change of diffuse beta-amyloid in amorphous deposits into grown-up amyloid filaments. For example astrocyte production of beta-amyloid and pathological chaperones such as ACT and apoE speed up the polymerization of betaamyloid peptides into filaments. Extracellular matrix proteins may become constant the amyloid structures. Finally neurons of the human brain respond to the glial activation and the mature amyloid deposits by tangle configuration, subsequent neuritic degeneration and ultimately cell death (Nilson et al., 1998).

\section{Genetics of Alzheimer Disease:}

There are three genes well-known to be important in the etiology of the early-onset familial condition: the APP on chromosome 21, the presenilin-1 (PS1) gene on chromosome 14, and the presenilin-2 (PS2) gene on chromosome 1. Apolipoprotein $\mathrm{E}$ on chromosome 19 is an important risk feature for sporadic Alzheimer disease.

\section{A. APP mutations}

The chief protein component of the extracellular plaque is $\beta$-amyloid. Soluble $\beta$-amyloid is a normal constituent of human brain generated by cleavage of the larger APP by two enzymes called $\beta$-secretase and $\gamma$-secretase. An alternative proteolytic pathway involving $\alpha$-secretase prevents A $\beta$ formation. $\beta$ -
Amyloid in the brain is heterogeneous, consisting of a series of peptides varying in length from 39 to 43 amino acids. $\beta$ - Amyloid of size 40 amino acids is referred to as $A \beta 40$, and is normally the most abundant form. $A \beta 42$ and $A \beta 43$ refer to the 42 and 43 amino acid forms, and the proportions of these two forms increase in the amyloid plaques of Alzheimer disease intelligence. Mutations in the APP that are known to cause some forms of autosomal dominant Alzheimer disease appear to alter normal APP handing out by causing greater than before production of $A \beta 42$ and $A \beta 43$. Another type of APP abnormality occurs in patients with Down syndrome, a condition caused by an extra copy of fraction or all of chromosome. Patients with Down syndrome are intellectually impaired and have a number of developmental abnormalities noted early in life. In mid adulthood, many go on to expand dementia with widespread declaration of $\beta$ amyloid in plaques and tangles similar to those seen in Alzheimer disease (Encyclopedia of Life Science, 2001).

\section{B. Presenilin mutations}

The most common known cause of familial Alzheimer disease is mutations in the presenilin genes. The presenilin proteins are transmembrane proteins that are localized first and foremost in the endoplasmic reticulum and the Golgi apparatus (Encyclopedia of Life Science, 2001). The majority and most antagonistic early onset cases are attributed to mutations of a gene located on chromosome 14, which produces a protein called presenilin 1. A structurally similar protein, presenilin 2, is produced by a gene on chromosome 1. Both presenilin 1 and presenilin 2 encode for membrane proteins that may be implicated in amyloid precursor protein (APP) processing. Scientists have identified more than 160 mutations in presenilin genes, and these mutations appear to result in abridged activity of $\gamma$-secretase, an enzyme important in $\beta$-amyloid peptide ( $\beta \mathrm{AP})$ formation (Dipiro et al., 2008).

\section{Apolipoprotein E e4 allele polymorphism}

Apo E is a major serum lipoprotein involved in cholesterol metabolism. There are three unsurprisingly occurring alleles of the ApoEgene, $\mathrm{e} 2$, e3 and e4, which differ from one another by a single codon (Encyclopedia of Life Science, 2001). Genetic susceptibility to sporadic, late-onset AD is thought to be primarily linked to the Apolipoprotein E (Apo E) genotype. The gene responsible for the production of apo $\mathrm{E}$ is located on chromosome 19. 
The mechanism through which Apo E4 confers an increased risk is unknown, although Apo E4 is associated with other factors that may contribute to AD pathology, such as abnormalities in mitochondria, cytoskeletal dysfunction, and low glucose usage. The quantity of risk depends on such factors as the number of Apo E4 copies, age, ethnicity, and gender. Overall, approximately $40 \%$ of patients with late-onset $\mathrm{AD}$ have at least one copy of Apo E4.

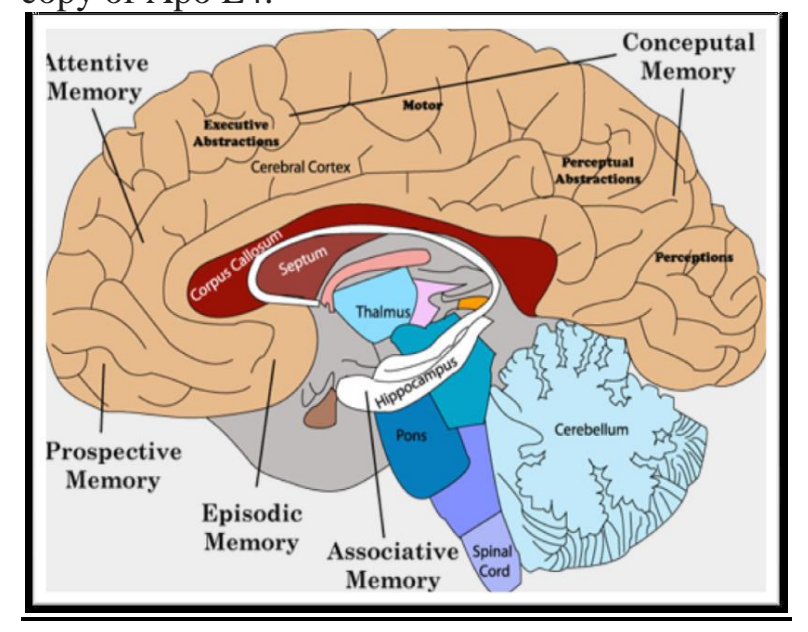

Figure No 1: Part of brain involved in different types of memory

Screening model for testing memory (Singh \& Parle, 2004):

A number of experiments are at present available for the screening of agents that adapt learning and memory processes. The models can be broadly classified as:

* Interoceptive behavior models (wherein aversive stimuli lie within the body)

* Exteroceptive behavior models (wherein aversive stimuli lie outside the body)

\section{Biochemical Markers:}

\section{Glutathione:}

The tri-peptide glutathione (g-glutamyl-cysteinylglycine) (GSH) is an endogenous antioxidant of great significance. Glutathione (GSH) is necessary for the upholding of the thiol redox status of the cell, protection against oxidative damage, detoxification of endogenous and exogenous reactive metal ions and electrophiles, storage and convey of cysteine, as well as protein and DNA synthesis, cell cycle regulation, and cell differentiation.

Glutathione and glutathione enzymes play a key responsibility in protecting the cell against the belongings of reactive oxygen species (ROS). The key functional element of glutathione is the cysteinyl moiety, which provides the reactive thiol group. ROS are reduced by GSH in the attendance of GSH peroxidase. As a result, GSH is oxidized to GSSG, which in turn is rapidly reduced back to GSH by GSSG reductase at the expense of NADPH.

In $\mathrm{AD}$, oxidized glutathione is greater than before while the activity of glutathione $\mathrm{S}$ transferase is decreased, consistent with the known augment in oxidative stress in AD (Butterfield et al., 2002).

\section{Malonaldehyde:}

Lipid peroxidation (LP) is distinct as the oxidative deterioration of lipid containing a number of carbon-carbon double bonds. A large quantity of toxic by-products is formed during LP. These have effects at a site away from area of their generation. Hence, they behave as toxic 'second messengers'. Membrane lipids are predominantly susceptible to LP.

Many of the products of LP are not overtly toxic or are minor products. Of major toxicological interest are Malonaldehyde (MDA), 4-hydroxynonenal (4$\mathrm{HNE}$ ), and various 2- alkenals (Devasagayam et al., 2003).

\section{Acetyl cholinesterase:}

Acetyl cholinesterase (AChE), one of the most indispensable enzymes in the family of serine hydrolases, catalyzes the hydrolysis of neurotransmitter acetylcholine, which plays a key position in memory and cognition. It has been elucidated that cholinergic deficiency is connected with $\mathrm{AD}$; therefore, one of the major beneficial strategies is to inhibit the biological activity of $\mathrm{AChE}$, and hence, to add to the acetylcholine level in the brain. Currently, most of the drugs used for the handling of AD are AChE inhibitors, as well as the synthetic compounds Tacrine, Donepezil, and Rivastigmine, which have all been proven to improve the state of affairs of AD patients to some extent (Lu et al., 2011).

\section{Treatment of Alzheimer's Disease:}

The main goal of treatment in $\mathrm{AD}$ is to symptomatically treat cognitive difficulties and protect patient occupation as long as possible. Secondary goals include treating the psychiatric and behavioral sequel that occurs as a result of the disease. 


\section{Non-pharmacologic treatment:}

$\mathrm{AD}$ has a reflective effect on both the patient and family, so appropriate treatment, both non pharmacologic and pharmacologic, is needed. Non medication interventions are the current primary interventions for supervision of AD.

Symptoms such as sleep disturbances, wandering, urinary incontinence, agitation, and hostility in patients with dementia are best managed by means of behavioral interventions rather than medications whenever possible. Communication between the patient and family members is essential in order to minimize stress on everyone.

Personal uneasiness may also trigger behaviors, so it is important to monitor for pain, hunger, thirst, constipation, full bladder, fatigue, infections and skin irritation, comfortable temperature, fears, and frustrations.

Creating a calm surroundings and removing stressors and triggers is a key. Education, communication, and planning are the key non pharmacologic components of caring for an $\mathrm{AD}$ patient. Preparation in the early stages of illness will lessen some of the caregiver constant worry as the illness progresses (Dipiro et al., 2008).

\section{Pharmacologic therapy}

While there is no cure for $\mathrm{AD}$, there are five prescription drugs approved by the U.S. Food and Drug Administration (FDA) to extravagance its symptoms.

Donepezil, Galantamine, Rivastigmine, and Tacrine are called "Cholinesterase Inhibitors". These drugs put off the breakdown of a chemical courier in the brain important for learning and memory. The fifth drug, Memantine, regulates the commotion of a different chemical courier in the brain that is also important for learning and memory (Narwal et al., 2012)

\section{Conclusion}

It is anticipated that nearly three fourths of the herbal drugs used wide-reaching were discovered following leads from local medicine. According to WHO about $25 \%$ of current medicines are descended from plants first used traditionally. Many others are synthetic analogues built on model compounds isolated from plants. Almost, 70\% modern medicines in India are consequent from natural products.

The basic uses of plants in medicine will prolong in the future, as a source of beneficial agents, and as raw material base for the pulling out of semi- synthetic chemical compounds such as cosmetics, perfumes and food industries.

\section{References}

1. Alzheimer's and Dementia, Alzheimer's Disease Facts and Figures (2013), Alzheimer's Association, Chicago, 9(2), pp 6-7.

2. Bijlani, R. L., (2004), Understanding medical physiology, Jaypee Brothers Medical Publishers (P) Ltd., New Delhi, 3, pp 861-864.

3. Butterfield, D.A., Pocernich, C.B., Drake, J., (2002), Elevated glutathione as a therapeutic strategy in Alzheimer's disease, Drug Development Research, 56, pp 428-437.

4. Chatterjee, C.C., (1997), Human physiology, 10th edition, Medical Allied Agency, Calcutta, 5(2), pp 264.

5. Devasagayam, T.P.A., Boloor, K.K., Ramasarma, T., (2003), Methods for estimating lipid peroxidation: An analysis of merits and demerits, Indian Journal of Biochemistry \& Biophysics, 40, pp 300-308.

6. Dipiro, J.T., Talbert, R.L., Yee G.C., Matzke G.R., Wells, B.G., Posey, L.M., (2008), Pharmacotherapy: A Pathophysiologic Approach, Mc Graw Hill, 7th Edition, pp 10511063.

7. Encyclopedia of Life Science (2001), Nature Publishing Group, pp 3-4.

8. Guyton and Hall (2006), Text book of Medical Physiology, Saunders an imprint of Elsevier, 11, pp 714-727.

9. Ingole, S.R., Satyendra, K., Sharma, R., Sharma, S.S., (2008), Cognition Enhancer: Current Strategies and Future Perspectives, Current Research \& Information on Pharmaceutical Sciences, 9, pp 42-48.

10. Jain, A.K., (2007), Text book of Physiology, Avichal Publishing company, Sirmour (HP), 2(3), pp 1058-1073.

11. Joshi H, Parle M, Cholinergic basis of memorystrengthening effect of Foeniculum vulgare Linn., Journal of Medicinal Food, 2006 Fall;9(3):413-7.

12. Kanwal, A., Mehla, J., Kuncha, M., Naidu, V.G.M., (2010), Anti-Amnesic activity of Vitex negundo in Scopolamine induced Amnesia in Rats, Pharmacology and Pharmacy, 1, pp 1-8.

13. Lu S.H., Wu J.W., Liu H.L., et al. (2011). The Discovery of potential acetylcholinesterase inhibitors: A Combination of pharmacophore modeling, virtual screening, and molecular docking studies, Journal of Biomedical Science, 18(8), pp 1-13. 
14. Narwal, S., Saini, D.R., Kumari, K., Narwal, S., Singh, G., Negi, R.S, Sarin, R.V., (2012), Behavior and Pharmacological Animal Models for the Evaluation of Learning and Memory Condition, Indo Global Journal of Pharmaceutical Science, 2(2), pp 121-129.

15. Nilson, L., Rogers, J., Potter, H., (1998), the Essential Role of Inflammation and Induced Gene Expression in the Pathogenic Pathway of Alzheimer's disease, Frontiers in Bioscience, 3, pp 436-446.

16. Oddo, S., Laferla, F.M., (2006), The Role of Nicotinic Acetylcholine Receptors in Alzheimer's disease, Journal of physiology, 99(2-3), pp 172-179.

17. Scahill, R.I., Schott, J.M., Stevens, J.M., Rossor, M.N., Fox, N.C (2002), Mapping the Evaluation of Regional Atrophy in Alzheimer's disease: unbiased Analysis of Fluid Registered Serial MRI, Proceding of the national academy of sciences USA, 99(7), pp 4703-4707.
18. Shapira, J., Schlesinger, R., Cummings, J.L., (1986), Distinguishing Dementias, American Journal of Nursing, 86(6), pp 698-702.

19. Shivakumar, L., Shivaraj, G., Rao, N.V., Verma, R., (2011) Evaluation of nootropic activity of polyherbal formulation SR-105 in experimental animals, International Research Journal of Pharmacy, 2(4), pp 101-107.

20. Singh, P., Parle, M., (2004), Animal models for testing memory, Asia Pacific Journal of Pharmacology, 16, pp 101-120.

21. Tripathi, J.K., (2006), Cognitive Deficits in Psychiatric Disorders: Current status, Indian Journal of Psychiatric, 48, pp 10-20.

22. Windhorst, G.R., (1996), Comprehensive Human Physiology, Springer-Verleg Berlin Heidelberg, 1, pp 1161-1162.

23. Zola, M.S, Squire, L.R., (1990), The Neuropshychology of Memory, Parallel findings in Humans and Non Human Primates, Ann NY Acad Sci, 608, pp 434-450. 